\title{
Una alternativa en la enseñanza de las ciencias
}

\author{
Humberto Caicedo López, Ms.Sc. \\ Profesor Titular \\ Departamento de Química \\ Universidad Pedagógica Nacional
}

Por años se ha discutido la importancia que se debe dar a los contenidos en contraposición a la de los procesos en la enseñanza de las ciencias. Se ha argumentado si a lo que se debe dar prioridad en la escuela es al aprendizaje del conocimiento acumulado en las ciencias o se debe dar mayor importancia al uso de los métodos que utilizan los científicos en la producción del conocimiento.

En efecto, la enseñanza de las ciencias en la última centuria se ha caracterizado más por dar importancia al aprendizaje de hechos, a la memorización de leyes y principios, con lo que no solo se ha desaprovechado la oportunidad de utilizar el aprendizaje de la ciencia como un evento educativo que contribuya mas eficazmente al desarrollo del individuo, sino que ha convertido el estudio de la ciencia en la escuela en una tarea estéril de memorización de información, generalmente sin sentido y sin utilidad, que de paso, proporciona una imagen distorsionada del trabajo científico y de la ciencia.

Es de todos conocido, también, que en los años sesenta se produce un movimiento significativo tendiente a orientar la enseñanza de las ciencias hacia el uso de los procesos, en los que se da prioridad a la naturaleza y la metodología del trabajo y la investigación científica. Aparece una gran cantidad de proyectos basados casi todos en el "aprendizaje por descubrimiento" y en el uso del "método científico" como herramienta de trabajo en el aula de clase. Sin embargo, estudios cuidadosos realizados, entre otros por Hodson(1982) y Ausubel(1986), sobre los resultados obtenidos con los nuevos currículos en lo que se refiere al aprendizaje logrado por los alumnos y en relación con la imagen de ciencia y de la investigación científica transmitida, permitieron concluir que existe todavía, entre profesores y alumnos, una concepción sobre la naturaleza de la metodología científica marcada por el inductivismo, con ignorancia de los aportes de las ideas de la epistemología contemporánea; que la visión del método único infravaloró el papel del trabajo, científico y llevó a los alumnos hacia una concepción de la ciencia que consiste en verdades absolutas, con que se introduce la rigidez y la intolerancia contra nuevas ideas; que el énfasis en el aprendizaje por descubrimiento, en donde se da excesiva importancia al valor motivacional de la experiencia directa, convirtió en un modelo de aprendizaje basado en concepciones empírico-inductivistas de la ciencia distorsionando la visión y la comprensión de la naturaleza la metodología científica. Al respecto concluye Ausubel, "en realidad con este procedimiento aprendieron poco la materia y menos aún el método científico".

Parece ser que la situación actual de la enseñanza de las ciencias en la escuela no ha variado en relación con lo descrito anteriormente. Diversos estudios recientes han concluido en que la mayoría de los cursos de los currículos de ciencias centran su interés en mayor grado en la transmisión y asimilación de hechos de la ciencia (Novak 1988). Otros estudios concluyen que muchas de las críticas a los modelos aprendizaje por descubrimiento desembocaron propuestas que reivindican modelos de transmisión asimilación de conocimientos ya elaborados, pues en ellos, de lo que se trata es de 
"aprender" ciencia, no de reconstruir o rehacer la ciencia. Con tal orientación obvio que la solución a los principales problemas que se han planteado sobre la enseñanza y el aprendizaje la ciencia queda todavía pendiente.

Ante esta situación lo que cabe preguntar es si existen hoy nuevas bases teóricas y metodológicas que den lugar a nuevas alternativas para el mejoramiento de la enseñanza de las ciencias en la escuela. La propuesta es, por supuesto, afirmativa. Los conocimientos y las ideas nuevas de la sicología cognitiva sobre el aprendizaje y las características del aprendiz, la emergencia de nuevas aproximaciones epistemológicas sobre la naturaleza de la construcción del conocimiento y las nuevas estrategias instruccionales que de éstas y aquéllas se derivan tendientes a capacitar al maestro para que apoye a sus estudiantes a - aprender a aprender', han generado ya y pueden seguir generando hacia el futuro proyectos nuevos que mejoren los currículos y los métodos de enseñanza de las ciencias. Es más, me atrevería a decir, que los argumentos sobre el énfasis que se debe dar a los contenidos o a los procesos, podrían diluirse si las nuevas ideas penetran con más fuerza en los centros de formación de maestros y se constituyen en parte esencial de los programas de capacitación de los maestros en servicio.

Existe hoy lo que Linn (1987> llama un "consenso emergente" entre filósofos, sicólogos y educadores que está sirviendo de guía para la planeación de los currículos de ciencias y para la formulación de estrategias de enseñanza. Este consenso incluye en primer lugar el alejamiento de las "leyes del aprendizaje» preconizadas por la sicología asociacionista que ignora la importancia y el rol que desempeña la afectividad en el aprendizaje escolar y la influencia positiva y negativa que ejercen los conocimientos que ya poseen los estudiantes en la adquisición de nuevos conocimientos.

En lo psicológico el consenso reconoce la importancia que tienen las concepciones alternativas que sostienen los estudiantes en la dirección de sus aprendizajes. Reconoce también la necesidad de estimular a los estudiantes para que se responsabilicen de su propia reorganización y elaboración conceptual, percatándolos del poder que poseen de aprender, de usar eficientemente lo aprendido y de establecer nuevas interrelaciones conceptuales. El uso de estas ideas junto con instrumentos apropiados ya han comenzado a probar que son capaces de crear condiciones para aprendizajes significativos, para desarrollar la creatividad y para generar actitudes positivas hacia la ciencia,

En lo epistemológico el consenso se refiere a que el individuo construye su propia visión de la ciencia y su concepción del mundo, a diferencia de las ideas dominantes por siglos de que la verdad de cómo funciona la naturaleza está fuera y por lo tanto el objetivo de la ciencia es la de "descubrir" las leyes de la naturaleza a través de la observación y la experimentación. Que así como el individuo construye su propia visión de la ciencia, los científicos como individuos trabajan con estas concepciones y construyen y elaboran nuevas ideas con las cuales explican las observaciones y los resultados de sus experiencias. Que además, las creencias, emociones y sentimientos juegan papel importante en la construcción del conocimiento con lo que se reconoce la importancia de lo que algunos Ilaman la "pasión» por la ciencia (Polyani,1956), tantas veces rechazada como elemento esencial en la construcción de conocimientos, por espíritus en lo que prevalecen posturas positivistas.

La teoría instruccional que se deriva de esta nueva visión de la ciencia, su enseñanza y su aprendizaje es por consiguiente sensiblemente diferente a la tradicional. Son también diferentes los papeles que se le asignan a los elementos claves de la educación: el 
estudiante, el maestro, el currículo, el contexto social y la evaluación. Es una perspectiva instruccional más optimista sobre la capacidad del individuo para aprender autónomamente y para construir su propio conocimiento, en donde son importantes tanto los conocimientos que ya posee el aprendiz, como sus concepciones sobre la. ciencia y el mundo; la responsabilidad del maestro es la de servir de guía y de tutor, compartir sus propios significados con sus alumnos y ayudarlos a aprender a generar su propio conocimiento; la planeación de los contenidos curriculares da más importancia a lo conceptual que a la descripción de hechos de la ciencia y tiene en cuenta la naturaleza de la construcción del conocimiento; la evaluación está más interesada en identificar problemas conceptuales que la cantidad de información retenida, así como a incentivar a los estudiantes a responsabilizarse de su propio aprendizaje.

Lo anterior exige, eso sí, nuevos métodos de enseñanza y el empleo de herramientas metacognitivas que le faciliten al estudiante aprender a generar su propio conocimiento, así como aprender cómo aprender. Por fortuna, están en vía de consolisidación instrumentos metacognitivos como el Mapa Cónceptual y el Diagrama Heurístico de Gowin (V Heurística) que se revelan prometedores a juzgar por los resultados obtenidos en varios programas de investigación (Nóvak, Gowin, 1984). Estos instrumentos que participan de la esencia de los principios de la sicología cognitiva sobre el aprendizaje significativo y de las ideas de la epistemología contemporánea ayudan a profesores y estudiantes a entender cómo se aprende significativamente y cómo se crea o se construye el conocimiento. En efecto, el mapa conceptual representa las relaciones entre los conceptos en forma jerárquica. Se usa como indicador de la organización cognitiva, para reconocer y entender la forma como un estudiante se desarrolla hacia un conocimiento experto y para evidenciar el grado como se está produciendo aprendizaje significativo por los tipos de diferenciaciones progresivas, reconciliaciones integradoras que se manifiestan cuando se construyen organizaciones conceptuales jerárquicas. El diagrama heurístico, diseñado inicialmente para ayudar a estudiantes y profesores a aclarar la naturaleza y propósitos del trabajo de laboratorio en ciencias, integra elementos teóricos y conceptuales con elementos metodológicos esenciales y permite además entender la construcción y naturaleza del conocimiento científico y el papel que desempeñan los conceptos en la observación e interpretación de los fenómenos.

De lo expuesto hasta aquí es evidente que el debate sobre la prioridad de los contenidos o procesos en los currículos de ciencia es innecesario pues ya no sería razonable enseñar los procesos de construcción del conocimiento sin enseñar simultáneamente los conceptos y los principios de la ciencia. Cualquier intento de enseñar los contenidos de la ciencia que no tenga en cuenta la compleja red conceptual que explica la construcción del conocimiento está destinado a fallar, si lo que se intenta con la ciencia en la escuela es provocar eventos educativos que involucren aprendizajes significativos.

El verdadero peligro estaría pues, en los currículos y en una instrucción que ignore la productiva simbiosis entre lo sicológico y lo epistemológico. Que ignore la síntesis de los dos consensos emergentes: de la sicología, que señala el papel fundamental desempeñan los conceptos en la adquisición del conocimiento y el de la epistemología, que caracteriza al conocimiento como una estructura evolutiva de conceptos y proposiciones. 


\section{REFERENCIAS BIBLIOGRAFICAS}

AUSUBEL, D (y otros) (1986) Educational Psycology: ACognitive View. Warbel an Peck.N.Y.

HODSON D.

LINN, M.

(1982) la there a Scientific Method? Education in chemistry July $112-116$

(1987) Establishing a Research Base for Science Education. Journal of Research in Science Teacher. 24(3), 191-216.

NOVAK, J.D.

(1988) "Human Constructivism: Toward a Unity of Psycological and Epistemological Meaning Making. Dep. of Educ. Cornell University Ithaca N.Y.

NOVAK, J.D. Gowin D.B. (1984) Learning How to Learn. Cambridge University press.

POLYANI, M.

(1956) Passion and Controversy in Science. The Lancet. 270921-925. 